Available online at www.easdprocedia.org

EASD Procedia EURODYN (2020) 2798-2806

EASD

Procedia
EURODYN 2020

XI International Conference on Structural Dynamics M. Papadrakakis, M. Fragiadakis, C. Papadimitriou (eds.) Athens, Greece, 23-26 November 2020

\title{
A 2.5D FEM-BEM-MFS METHODOLOGY FOR SOIL-STRUCTURE INTERACTION PROBLEMS IN LAYERED HALF-SPACES
}

\author{
Hassan Liravi ${ }^{1}$, Robert Arcos ${ }^{1,2}$, Dhananjay Ghangale ${ }^{1}$, and Jordi Romeu ${ }^{1}$ \\ ${ }^{1}$ Acoustical and Mechanical Engineering Laboratory (LEAM), Universitat Politècnica de Catalunya \\ (UPC). \\ c/ Colom, 11, 08222 Terrassa (Barcelona), Spain. \\ e-mail: \{hassan.liravi,dhananjay.ghangale,jordi.romeu\}@upc.edu \\ ${ }^{2}$ Serra Húnter Fellow, Universitat Politècnica de Catalunya (UPC). \\ c/ Colom, 11, 08222 Terrassa (Barcelona), Spain. \\ e-mail: robert.arcos@upc.edu
}

Keywords: Method of fundamental solutions, elastodynamics, soil-structure interaction, railway tunnels, layered half-space.

Abstract. In this paper, a new prediction methodology to deal with longitudinally invariant soil-structure interaction problems in elastodynamics is presented. The method uses the finiteelement method to model the structure, the boundary-element method to model the local soil surrounding the structure and the method of fundamental solutions to model the wave propagation through the soil. All those methods are formulated in the two-and-a-half-dimensional domain. The methodology is firstly verified in the framework of a homogeneous half-space system by comparing the results of the current method with those computed by the semi-analytical solution of this problem. Secondly, the methodology is verified against a two-and-a-half-dimensional finite element-boundary element approach, for two calculation examples: a tunnel embedded in a homogeneous half-space and a tunnel embedded in a layered half-space. This comparison also shows that this novel methodology reduces the computational costs of such simulations without compromising the accuracy of the results. The increase on the computational efficiency is due to the use of the method of fundamental solutions to account for the wave propagation in the medium, and it is even higher when the number of evaluations points increases. 


\section{INTRODUCTION}

In the framework of soil-structure interaction problems, some structures can be considered as longitudinally invariant systems that can be assessed by two-and-half-dimensional approaches. Structures like at-grade railway tracks [4], tunnels [1], roads [2] or bridges [3] are some examples of soil-structure problems where the structure can be modelled as longitudinally invariant system. A review of the literature indicates that there are several works that contributed to the study of this kind of soil-structure interaction problems. Among them, coupled 2.5D finite element-boundary element methodology (2.5D FEM-BEM) is a well-known numerical approach for the assessment of the soil-structure interaction problems. François et al. [2] presented a 2.5D FEM-BEM model in frequency domain. They proposed this method to study the wave propagation in soil induced by railway or road traffic using this method. Galvín et al. [4] used the same method to analyse two different applications: a ballasted track over a layered half-space and a tunnel embedded in a layered half-space. Lopes et al. [5] proposed an alternative method where a $2.5 \mathrm{D}$ FEM with 2.5D perfect matched layers (PML) is used to assess the ground-borne vibration response of a building. Later, the re-radiated noise inside the building was investigated using a 2.5D MFS in acoustics weakly coupled with the building vibration field obtained with the method presented in [5] [6]. More recently, Ghangale and his colleagues [7] presented a method for the prediction of the energy flow radiated by underground railway infrastructures based on a 2.5D FEM-BEM method to model the tunnel and the locally surrounding ground and on the semi-analytical solutions of a cylindrical cavity to model the wave propagation on the soil.

The MFS is a collocation method which approximates the solution within an elastic medium employing a combination of fundamental solutions of the medium governing equations. It is specially interesting for dealing with wave propagation problems in unbounded or partially unbounded domains. Its application is based on a distribution of collocation points, which evaluates the boundary conditions at discrete positions, and on a distribution of source points (or virtual forces, in elastodynamic problems), which are obtained by complying the boundary conditions at the collocation points. The collocation points are located on the boundary and the source points outside the domain. Godinho et al. [11] presented two-dimensional FEM-MFS modelling approach for these types of problems. An extension of this to longitudinally invariant systems was presented by Amado-Mendes and his colleagues [1], where a methodology that models the structure using 2.5D FEM and the surrounding soil with 2.5D MFS is proposed. For many years, it has been a concern of the researchers to find the optimal distance between collocation and source points, particularly in complicated shapes. An incorrect selection of this distance may lead to large errors of the numerical method [9]. Besides, it should be noted that the amount of source points affects the numerical convergence and stability of the results [10].

In this study, a 2.5D FEM-BEM-MFS method in frequency domain is presented. The $2.5 \mathrm{D}$ FEM-BEM is used to obtain the displacement field on the boundary and the 2.5D MFS is used as a post-processing tool to obtain the displacement and traction fields on the soil from the displacement field on the soil-structure interaction boundary. The main novelty of the current method is, thus, the way MFS is applied, which leads to two global benefits. On the one hand, the application of this methodology results to an increase of the computational efficiency of the method respect to traditional 2.5D FEM-BEM approaches and this is mostly significant when many evaluator points are to be analysed. On the other hand, a significant difference between the work of Amado-Mendes et al. [1] and the current method is related about how the dynamic stiffness matrix of the soil at the soil-structure interaction boundary is obtained. 


\section{Description of the methodology}

In this study, the calculations are performed in two general steps. Firstly, the displacements on the boundary are computed through a 2.5D FEM-BEM method. In this 2.5D FEM-BEM approach, the FEM is used to model the structure and the BEM is used to model the locally surrounding soil. The formulation of the 2.5D FEM-BEM used here is based on [2]. The response of the soil-structure system can be calculated by

$$
\left[\mathbf{K}_{0}-\mathbf{i} k_{x} \mathbf{K}_{1}+k_{x}{ }^{2} \mathbf{K}_{2}+\overline{\mathbf{K}}_{s}-\omega^{2} \mathbf{M}\right] \overline{\boldsymbol{U}}=\overline{\boldsymbol{F}},
$$

where $\mathbf{K}_{0}, \mathbf{K}_{1}, \mathbf{K}_{2}$ and $\mathbf{M}$ are the stiffness and mass matrices related to the 2.5D FEM domain, $\overline{\mathbf{K}}_{s}$ is the dynamic stiffness matrix of the soil obtained by $2.5 \mathrm{D}$ BEM model, $k_{x}$ is the longitudinal wavenumber and $\omega$ is the angular frequency. Moreover, $\overline{\boldsymbol{U}}$ and $\overline{\boldsymbol{F}}$ are the vectors of displacements and applied external forces along the mesh of the structure, respectively. Thus, the displacements $\bar{U}_{b}$ on the boundary can be extracted from $\bar{U}$.

Secondly, the displacement and traction fields in the soil are calculated using a 2.5D MFS for elastodynamics. For this aim, the displacements on the boundary $\left(\overline{\boldsymbol{U}}_{\boldsymbol{b}}\right)$ are considered as the boundary conditions of the MFS. Then, the virtual source strengths can be computed as

$$
\overline{\boldsymbol{S}}_{v}=\overline{\mathbf{H}}_{c s}^{-1} \overline{\boldsymbol{U}}_{c}
$$

where $\overline{\boldsymbol{S}}_{v}$ is the vector of virtual source strengths, $\overline{\mathbf{H}}_{c s}$ represents the matrix of displacement Green's functions on the collocation points due to the virtual sources and $\overline{\boldsymbol{U}}_{c}$ represents the displacements at the collocation points. If the collocation points are considered to be directly the nodes of boundary mesh, $\overline{\boldsymbol{U}}_{c}=\overline{\boldsymbol{U}}_{b}$. If the configuration is different, the displacement in the collocation points $\bar{U}_{c}$ should be obtained by interpolation along the boundary from $\overline{\boldsymbol{U}}_{b}$. In this study, the Green's function are computed by the ElastoDynamics Toolbox for MATLAB [8]. Given the source strengths, the displacement and traction fields can be calculated as

$$
\overline{\boldsymbol{U}}_{f}=\overline{\mathbf{H}}_{f s} \overline{\boldsymbol{S}}_{v}, \quad \overline{\boldsymbol{T}}_{f}=\overline{\mathbf{H}}_{f s}^{\tau} \overline{\boldsymbol{S}}_{v}
$$

where $\overline{\mathbf{H}}_{f s}$ and $\overline{\mathbf{H}}_{f s}^{\tau}$ represent the source-evaluators Green's functions for displacements and tractions, respectively, and $\bar{U}_{f}$ and $\overline{\boldsymbol{T}}_{f}$ stand for the displacement and traction of the evaluation points, inside the domain.

In order to improve the computational efficiency of the method, a mapping technique is used. In this technique, the Green's displacement and Green's traction fields are calculated for the unique values of the sources depth and relative distances of the source-receiver points. Then, these Green's functions are mapped into the original configuration of sources and evaluation points by performing the required coordinate rotations [7].

\section{Verification of the method for a homogeneous half-space problem}

The proposed method is firstly verified for a solid cylinder with a radius of $3 \mathrm{~m}$ embedded in a homogeneous half-space, a system illustrated in Fig. 1. In order to compare with the semianalytical solutions of a homogeneous half-space for verification purposes, the solid cylinder is assumed to be defined by the exact same mechanical parameters than the soil. The mechanical parameters of the soil are defined in Table 1. The vertical displacements obtained with the new 
method are plotted against the results obtained by the semi-analytical solution of the problem and by the 2.5D FEM-BEM approach for a wavenumber of $0.1 \mathrm{rad} / \mathrm{m}$. Results of this verification are shown in terms of displacement Green's functions in the wavenumber-frequency domain, presented in $\mathrm{dB}$ based on reference of $10^{-12} \mathrm{~m} /(\mathrm{N} / \mathrm{m})$.

\begin{tabular}{ccccc}
\hline Type & $E[\mathrm{MPa}]$ & $\rho\left[\mathrm{kg} / \mathrm{m}^{3}\right]$ & $\nu$ & Damping \\
\hline Cylinder & 108 & 1800 & $1 / 3$ & 0.05 \\
Soil & 108 & 1800 & $1 / 3$ & 0.05 \\
\hline
\end{tabular}

Table 1: Mechanical parameters of the solid cylinder and the soil.

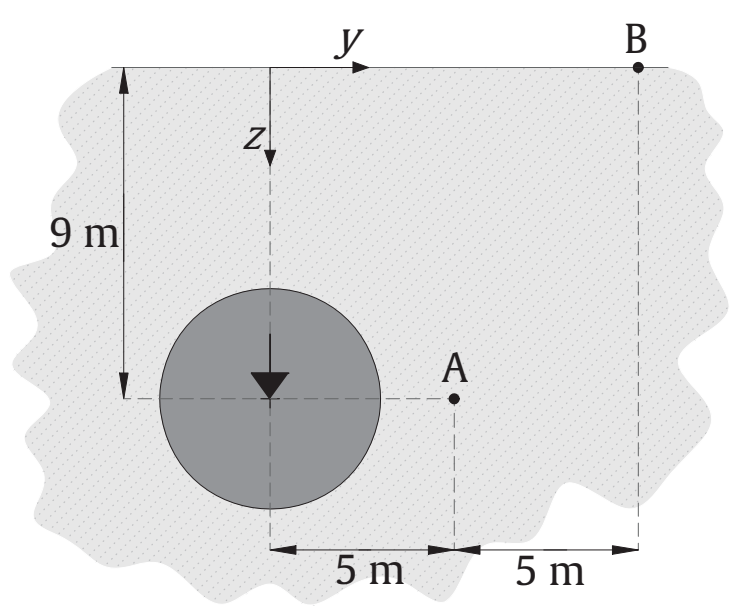

Figure 1: Geometry of the system studied, based on a solid cylinder in a homogeneous half-space. A and B represent the field points on the soil where the results will be obtained. The input vertical force applied at the center of the cylinder is represented by a big arrow.

According to the results presented in Fig. 2, very good agreement of the results is observed between the three methods compared. The results of the current method and those obtained by 2.5D FEM-BEM method are almost exactly matched. However, a slight difference can be observed between the results of the proposed method and semi-analytical solution, particularly at frequencies higher than $60 \mathrm{~Hz}$, due to the mesh size. 
(a)

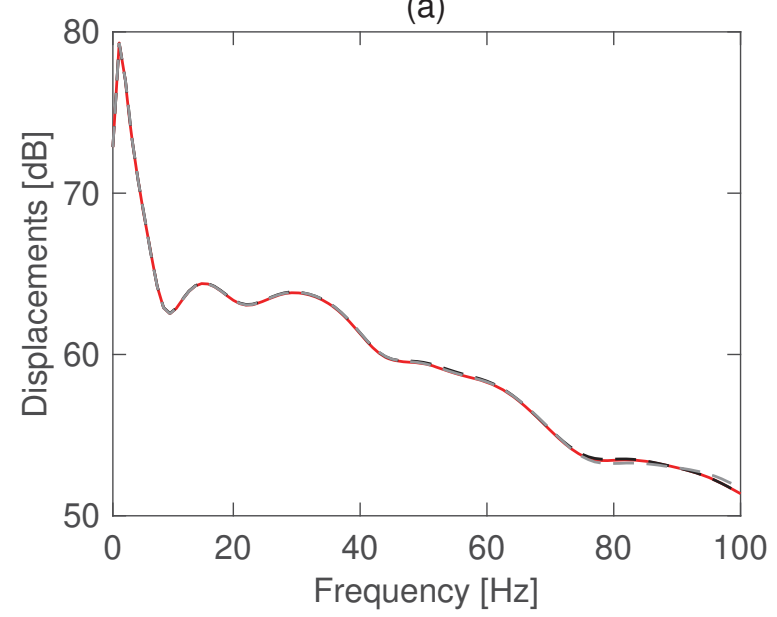

(b)

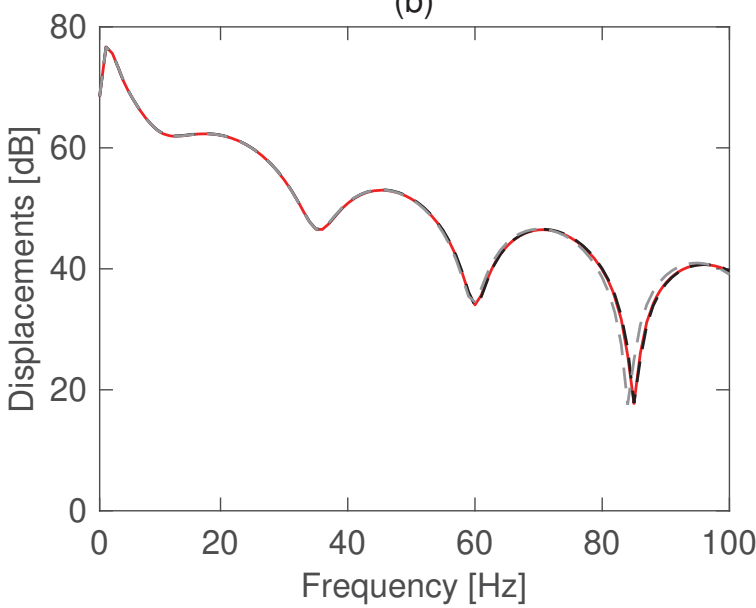

Figure 2: Vertical displacement Green's functions at evaluation points A (a) and B (b). Methods: 2.5D FEM-BEM (solid red line), 2.5D FEM-BEM-MFS (dashed black line) and semi-analytical solution (dashed gray line).

\section{Verification of the method for the case of a tunnel embedded in a half-space}

In this section, the methodology is verified against a 2.5D FEM-BEM approach, for two calculation examples: a tunnel embedded in a homogeneous half-space and a tunnel embedded in a layered half-space. In these examples, the comparison is done in terms of the displacement Green's function due to a vertical force applied in the bottom of the tunnel and for the wavenumber of $0.1 \mathrm{rad} / \mathrm{m}$. The mechanical parameters of the soil and tunnel are presented in Table 2 and Table 3 for the homogeneous and layered half-space cases, respectively. In the example of a tunnel embedded in a layered half-space, the soil is divided into three layers.

\begin{tabular}{ccccc}
\hline Type & $E[\mathrm{MPa}]$ & $\rho\left[\mathrm{kg} / \mathrm{m}^{3}\right]$ & $\nu$ & Damping \\
\hline Tunnel & 31000 & 2500 & 0.2 & 0.001 \\
Medium & 108 & 1800 & $1 / 3$ & 0.05 \\
\hline
\end{tabular}

Table 2: Mechanical parameters of the tunnel and the soil for the homogeneous half-space case.

\begin{tabular}{cccccc}
\hline Type & $E[\mathrm{MPa}]$ & $\rho\left[\mathrm{kg} / \mathrm{m}^{3}\right]$ & $\nu$ & Thickness $[\mathrm{m}]$ & Damping \\
\hline Tunnel & 31000 & 2500 & 0.2 & - & 0.001 \\
Soil layer 1 & 50 & 1900 & 0.3 & 4 & 0.05 \\
Soil layer 2 & 180 & 1980 & 0.3 & 9 & 0.05 \\
Soil layer 3 & 400 & 2050 & 0.3 & $\infty$ & 0.05 \\
\hline
\end{tabular}

Table 3: Mechanical parameters of the tunnel and the soil for the layered half-space case.

\subsection{A tunnel embedded in a homogeneous half-space}

The application of the methodology for a tunnel embedded in a homogeneous half-space is studied. As shown in Fig. 3, a tunnel embedded in a homogeneous half-space at a depth of $9 \mathrm{~m}$ 
from the ground surface is assumed. The external radius and the lining thickness of the tunnel are $3 \mathrm{~m}$ and $0.3 \mathrm{~m}$, respectively. A single vertical point load is considered to be acting on the bottom surface of the tunnel. Two evaluation points, one on the near field (A), one in the far field (B) are assumed in the soil. The vertical displacements obtained by the proposed methodology are compared with those obtained by the 2.5D FEM-BEM approach. This comparison is shown in Fig. 4, where the left graph represents the vertical displacements in the point $\mathrm{A}$ and right figure denotes the vertical displacements at point B.

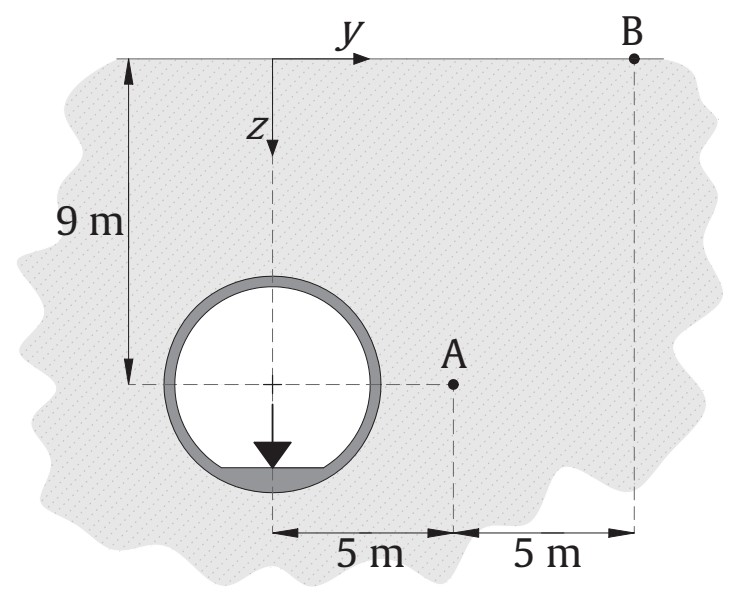

Figure 3: Geometry of the second system studied, based on a tunnel embedded in a homogeneous half-space. A and B represent the field points on the soil where the results will be obtained. The input vertical force is represented by a big arrow.

As presented in Fig. 4, the vertical displacements obtained by the 2.5D FEM-BEM-MFS are in a very good agreement with respect to those obtained with the 2.5D FEM-BEM approach.

(a)

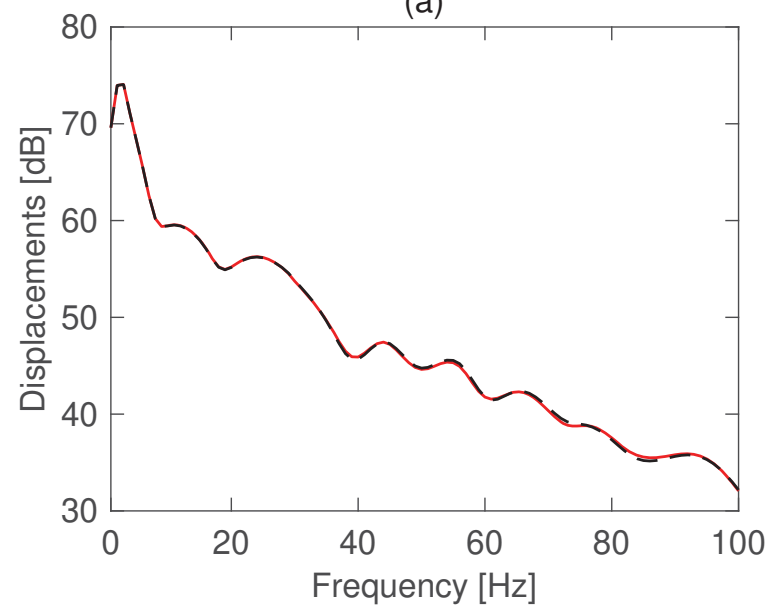

(b)

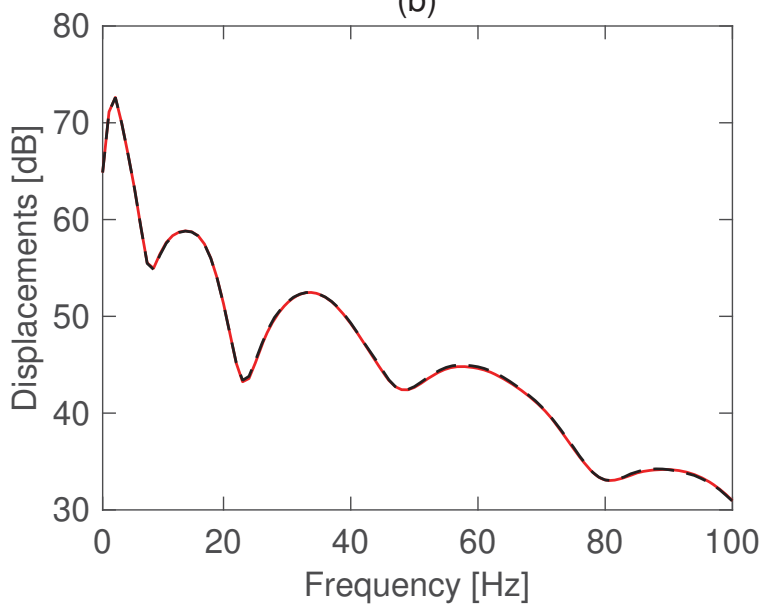

Figure 4: Vertical displacement Green's functions for the system in Fig. 3. Methods: 2.5D FEM-BEM (solid red line) and 2.5D FEM-BEM-MFS (dashed black line). 


\subsection{A tunnel embedded in a layered half-space}

In order to investigate the performance of the method for a tunnel embedded in a layered half-space, another example is presented in this section. As can be observed in Fig. 5, a tunnel structure is located in the second layer and two field points are assumed in the soil (A and B). The geometry of the tunnel is the same as previous example.

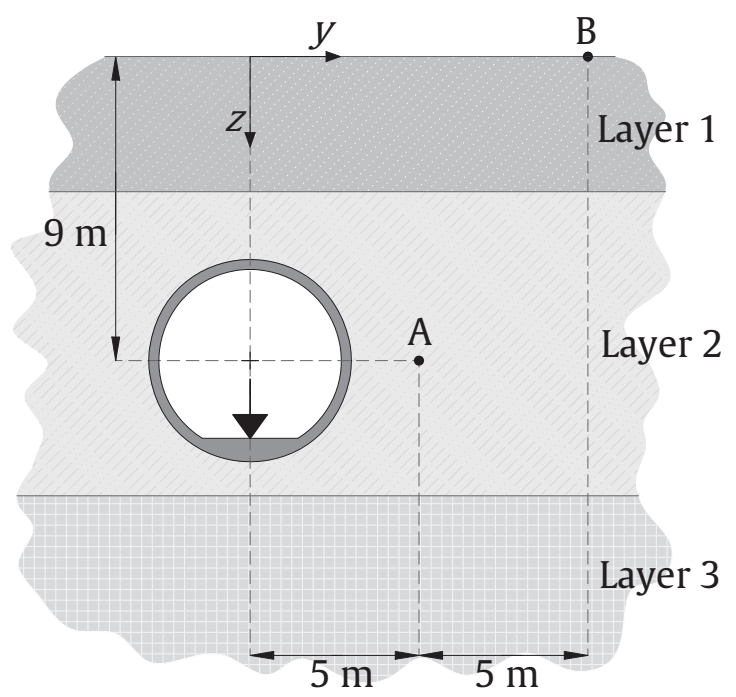

Figure 5: Geometry of the third system studied, based on a tunnel embedded in a layered half-space. A and B represent the field points on the soil where the results will be obtained. The input vertical force is represented by a big arrow.

The results confirms that the proposed methodology can be used for the case of layered half-space. Based on Fig. 6, the results of the current methodology are again consistent with the results of the 2.5D FEM-BEM approach. However, a slight difference can be observed at frequencies higher than $60 \mathrm{~Hz}$ for the point $\mathrm{A}$.

(a)

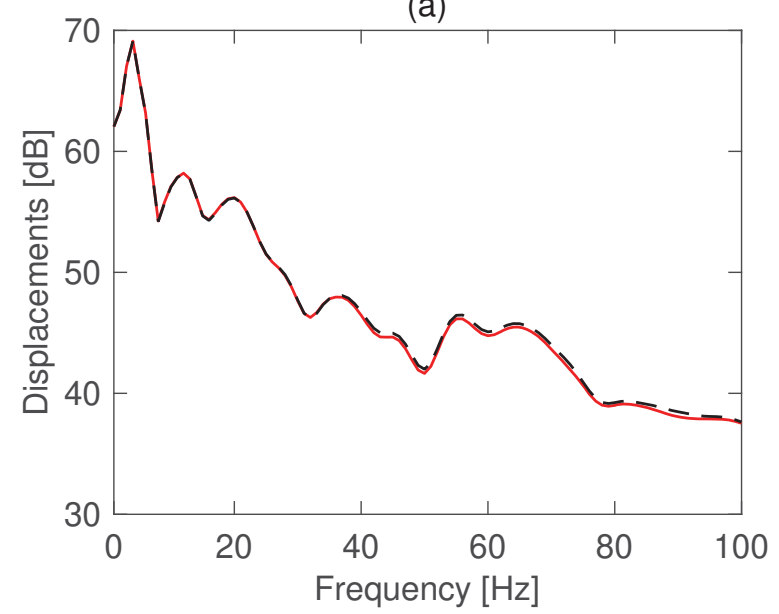

(b)

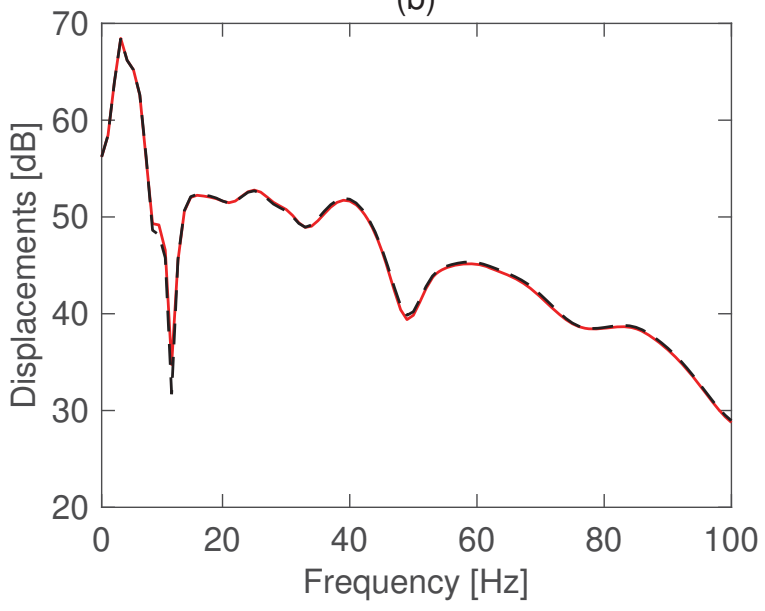

Figure 6: Vertical displacement Green's functions for the system in Fig. 5 at evaluation points A (a) and B (b). Methods: 2.5D FEM-BEM (solid red line) and 2.5D FEM-BEM-MFS (dashed black line). 


\section{CONCLUSIONS}

In this study, a methodology is presented to deal with longitudinally invariant soil-structure interaction problems in elastodynamics. The methodology is firstly verified for the case of a solid cylinder embedded in a homogeneous half-space, having the structure and the soil the same mechanical parameters. Then, the performance of the model is investigated by presenting two calculation examples; a tunnel embedded in a homogeneous half-space and a tunnel embedded in a layered half-space. The results obtained for the three cases studied confirm that the accuracy of the new 2.5D FEM-BEM-MFS method is in agreement with those obtained by 2.5D FEMBEM, especially in the far field results. Furthermore, the inclusion of the MFS as a postprocessing tool has found to be very simple to be implemented. However, it is also found, as was expected, that the accuracy of the 2.5D FEM-BEM-MFS strongly depends on the accuracy of the boundary conditions obtained by 2.5D FEM-BEM method. Moreover, the displacement and traction Green's functions in the field points can be obtained in $\mathrm{x}, \mathrm{y}$ and $\mathrm{z}$ directions. However, in this paper, only the vertical displacements are presented.

The accuracy of the results can be improved considerably by controlling the robustness of the method. To do so, a check point can be assumed in the domain and the distance between virtual sources and collocation points can be optimised by minimising the relative error between the current method and 2.5D FEM-BEM approach at this control point.

\section{REFERENCES}

[1] Amado-Mendes, P., Alves Costa, P., Godinho, L. M. C., Lopes, P., 2.5D MFS-FEM model for the prediction of vibrations due to underground railway traffic, Engineering Structures, 104, 141-154, 2015.

[2] François, S., Schevenels, M., Galvín, P., Lombaert, G., Degrande, G., A 2.5D coupled FE-BE methodology for the dynamic interaction between longitudinally invariant structures and a layered halfspace, Computer Methods in Applied Mechanics and Engineering, 199(23-24), 1536-1548, 2010.

[3] Tongaonkar, N. P., Jangid, R. S., Seismic response of isolated bridges with soil-structure interaction, Soil Dynamics and Earthquake Engineering, 23(4), 287-302, 2003.

[4] Galvín, P., François, S., Schevenels, M., Bongini, E., Degrande, G., Lombaert, G., A 2.5D coupled FE-BE model for the prediction of railway induced vibrations, Soil Dynamics and Earthquake Engineering, 30(12), 1500-1512, 2010.

[5] Lopes, P., Alves Costa, P., Ferraz M, Calçada, R. Cardoso, A. Silva., Numerical modeling of vibrations induced by railway traffic in tunnels: From the source to the nearby buildings, Soil Dynamics and Earthquake Engineering 61-62, 269-285, 2014.

[6] Colaço, A., Alves Costa, P., Amado-Mendes, P., Godinho, L., Calçada, R., Mitigation of vibrations and re-radiated noise in buildings generated by railway traffic: A parametric study, Procedia Engineering, 199, 2627-2632, 2017.

[7] Ghangale, D., Arcos, R., Clot, A., Cayero, J., Romeu, J., A methodology based on 2.5D FEM-BEM for the evaluation of the vibration energy flow radiated by underground railway infrastructures. Tunnelling and Underground Space Technology, 2020. 
[8] Schevenels, M., François, S., Degrande, G., EDT: An ElastoDynamics Toolbox for MATLAB, Computers and Geosciences, 35(8), 1752-1754, 2009.

[9] Alves, C. J. S., On the choice of source points in the method of fundamental solutions, Engineering Analysis with Boundary Elements, 33(12), 1348-1361, 2009.

[10] Sun, Y., Marin, L., An invariant method of fundamental solutions for two-dimensional isotropic linear elasticity, International Journal of Solids and Structures, 117, 191-207, 2017.

[11] Godinho, L., Amado-Mendes, P., Pereira, A., Soares, D., A coupled MFS-FEM model for 2-D dynamic soil-structure interaction in the frequency domain, Computers and Structures, 129, 74-85, 2013. 